\title{
Clinical assessment of oral mucositis and candidiasis compare to chemotherapic nadir in transplanted patients
}

\section{Cleverson PATUSSI (a) \\ Laurindo Moacir SASSI(b) \\ Eduardo Ciliao MUNHOZ(c) \\ Roberta Targa Stramandinoli \\ ZANICOTT!(b) \\ Juliana Lucena SCHUSSEL ${ }^{(b)}$}

\footnotetext{
(a) Department of Oral Medicine, Post Graduation Program in Dentistry, Universidade Federal do Paraná - UFPR, Cuitiba, PR, Brazil

(b)Department of Oral and Maxillofacial Surgery, Hospital Erasto Gaertner, Curitiba, PR, Brazil.

(c)Department of Bone Marrow Transplantation, Hospital Erasto Gaertner, Curitiba, PR, Brazil.
}

Declaration of Interests: The authors certify that they have no commercial or associative interest that represents a conflict of interest in connection with the manuscript.

Corresponding Author:

Cleverson Patussi

E-mail: cleversonpatussi@hotmail.com

DOI: 10.1590/1807-3107BOR-2014.vol28.0050

Submitted: Nov 12, 2013

Accepted for publication: Jun 02, 2014

Last revision: Aug 18, 2014

\begin{abstract}
Oral mucositis is a chief complication in patients undergoing hematopoietic stem cell transplantation (HSCT). It is considered a toxic inflammatory reaction that interferes with the patient's recuperation and quality of life. Oral candidiasis is a common fungal infection observed in dental practice, particularly in immunocompromised patients. The aim of this study was to evaluate the presence of oral mucositis and oral candidiasis in patients who underwent HSCT and their correlation with the chemotherapeutic nadir (lowest possible outcome). We evaluated patients with different diagnoses who underwent HSCT at the Hospital Erasto Gaertner. No chemotherapeutic nadir curves could be associated with mucositis, and patients had different presentations of mucositis. No patient developed oral candidiasis during hospitalization. Together with cell counts, we collected demographic data including age, oral hygiene, habits harmful to health, and the use of oral prostheses. It was observed that patients who smoked cigarettes before hospitalization showed less mucositis, resulting in no feeding problems or other comorbid conditions due to the effect of mucositis. However, the nadir of the chemotherapy curve, in isolation, is not a predictive tool for the appearance (or no appearance) of oral mucositis.
\end{abstract}

Keywords: Candidiasis; Mucositis; Bone Marrow Transplantation; Oral Medicine; Hematopoietic Stem Cell Transplantation.

\section{Introduction}

Oral mucositis is characterized by a toxic inflammatory reaction that can occur on exposure to chemotherapeutic agents and/or ionizing radiation. It is common during oncological therapy, and is the most common complication and cause of pain in the oral cavity in patients undergoing Hematopoietic Stem Cell Transplantation (HSCT), autologous or allogeneic, with a frequency of $75 \%-99 \%$. $^{1}$ This condition affects the mucosa of the gastrointestinal tract, which includes the oral cavity and oropharynx.

Oral candidosis is an opportunistic fungal infection mostly associated with local and systemic conditions such as immunosuppression. Lehner (1967) described different manifestations of oral candidiasis, based on clinical, bacteriological, histological, and therapeutic criteria as follows: pseudomembranous candidiasis, acute atrophic candidiasis, ulcerative stomatitis related to denture, angular cheilitis, chronic atrophic candidiasis, candidal leukoplakia, candidiasis associated with endocrine disorders, median rhomboid glos- 
sitis, and chronic diffuse candidiasis. ${ }^{2}$ This is the most accepted and most frequently used classification.

"Nadir" means the lowest point. Soon after chemotherapy infusion, bone marrow cells stop dividing, and the remaining cells are able to ensure blood stability for a few days. Between the 10 and 14 days after the start of chemotherapy, there is an intense decrease in cell count. This period is known as the chemotherapeutic nadir and represents the lowest point on the curve of blood cell counts. The term implies that cells will return to increase with time. ${ }^{3}$

Studies have shown that the occurrence of clinical mucositis occurs inversely to bone marrow function, being higher during the nadir of neutropenia and healing, culminating with the recovery of granulocytes. ${ }^{3,4}$ The aim of the present study was to evaluate the occurrence of mucositis and oral candidiasis in patients who underwent Hematopoietic Stem Cell transplantation in the Hospital Erasto Gaertner (HEG, Curitiba, Brazil), and to compare that occurrence with the chemotherapeutic nadir of these patients.

\section{Methodology}

For this study, we selected patients referred for HSCT as part of cancer treatment in 2012. The study was approved by the Committee on Ethics and Research at the Hospital Erasto Gaertner. All selected patients signed an informed consent to participate in the research. Data collected from their medical records included reported pain or complaints related to the oral cavity, and patient leukocyte and platelet levels during hospitalization.

Blood tests were performed according to medical criteria almost daily, with the phases of treatment taken into consideration.

Mucositis was evaluated according to the Oral Toxicity Scale of the World Health Organization (WHO), ${ }^{5}$ which is based on objective (redness or erythema, ulcer development) and subjective signs (ability to swallow, sensitivity of the mucosa) and classifies mucosa into four grades: Grade 0, no change observed in oral cavity during treatment; Grade I, presence of pain and erythema in mucosa, gums, tongue, or palate; Grade II, presence of erythema and ulcers, being able to perform normal feeding; Grade III, presence of ulcers, only "pasty" food supported; and Grade IV, presence of ulcers, erythema, and pain, rendering feeding impossible (Table 1).
Table 1. World Health Organization Oral Mucositis Classification

\begin{tabular}{lc}
\hline Mucositis - Oral Toxicity Scale (WHO) & \\
\hline Grade 0 & No alterations \\
Grade I & Pain and erythema \\
Grade II & Erythema and ulcers \\
Grade III & Ulcers (liquid diet only) \\
Grade IV & Unable to feed \\
\hline
\end{tabular}

Oral candidiasis assessment was based on the Lehner scale (Table 2$)^{2}$ for cohort standardization. Oral condition was evaluated daily, with data collected on the occurrence of mucositis and candidiasis.

Table 2. Lehner Oral Candidiasis Classification

\begin{tabular}{|c|c|c|}
\hline \multicolumn{3}{|l|}{ Lehner Rating } \\
\hline \multirow[t]{2}{*}{ Acute forms } & \multicolumn{2}{|c|}{ Pseudomembranous candidiasis } \\
\hline & \multicolumn{2}{|c|}{ Acute atrophic candidiasis } \\
\hline \multirow[t]{2}{*}{ Chronic forms } & Atrophic & $\begin{array}{l}\text { 1. ulcerative stomatitis related to denture } \\
\text { 2. angular cheilitis }\end{array}$ \\
\hline & Hyperplasic & $\begin{array}{l}\text { 1. chronic atrophic candidiasis } \\
\text { 2. candida leukoplakia } \\
\text { 3. candidiasis associated with } \\
\text { endocrine disorders } \\
\text { 4. median rhomboid glossitis } \\
\text { 5. chronic diffuse candidiasis. }\end{array}$ \\
\hline
\end{tabular}

Low-frequency laser applications were performed during hospitalization periods at least two times per week. Some patients presented severe mouth pain and feeding difficulties, and received extra low-frequency laser applications. All patients were instructed to use chlorhexidine $0.12 \%$ mouthwash, twice a day, to reduce and control oral biofilm and support oral hygiene during hospitalization.

Patients were classified into groups in accordance with study variables, such as tobacco use, diagnosis, type of transplant, oral hygiene, and use of oral prostheses. Those who had the same diagnosis underwent the same chemotherapy regimen, particularly if they had undergone the same type of transplant and were in the same variable-based group. In this way, the study was based on comparative data generated by the study population, with a series of inferences as discussed below.

\section{Results}

Thirty-one patients were prospectively selected from the Department of Hematopoietic Stem Cell 
Transplantation (HSCT), Hospital Erasto Gaertner, between January and December of 2012. Among the selected patients, 15 were male and 16 female, with an average age of 43.8 years (range, 19-66 years). The most frequent diagnosis was lymphoma (64\%), followed by multiple myeloma (19\%) and leukemia (12\%). One patient had metastatic embryonic carcinoma. During hospitalization, one patient died from the disease. In total, 28 patients underwent autologous transplant and three received allogeneic therapy (Table 3).

No patient developed candidiasis during hospitalization. Four patients (12\%) presented no mucositis, $15(48 \%)$ presented Grade I, five (16\%) presented Grade II, five (19\%) presented Grade III, and only one patient presented Grade IV.

Patients with lymphoma (50\%), multiple myeloma $(19 \%)$, and leukemia (13\%) presented mucositis Grades I, II, and III. Grade IV occurred in a patient with nonHodgkin's lymphoma. From the 20 patients diagnosed with lymphoma, 50\% had Hodgkin's and 50\% had non-Hodgkin's lymphoma.

The evaluation of the chemotherapeutic nadir takes into consideration the levels of leukocytes and platelets. Most patients showed a decrease in the number of leukocytes between days $\mathrm{D}+2$ and $\mathrm{D}+8$. The platelet nadirs were similar and started mostly on day D + 2; most patients left the hospital with platelet counts under 150,000 $\mathrm{mm}^{3}$. The platelet nadir curve showed instability due to platelet transfusion performed in patients with severe thrombocytopenia.

Oral biofilm assessment evaluated only its presence or absence. Of the 31 patients evaluated, 16 had visible biofilm on daily assessment, and 15 showed no visible biofilm. Dentures were not worn by $61 \%$ of patients, $26 \%$ were wearing at least one fixed denture, $10 \%$ used at least one removable partial denture, and $3 \%$ had implant-supported dentures. No association was found between the use of different types of dental prostheses and the occurrence of mucositis.

Patients who underwent autologous transplant showed various grades of mucositis, and those who underwent allogeneic therapy presented Grades I and III. No correlation was found between the type of transplant and the occurrence of mucositis.
Smokers constituted $29 \%$ of patients, but all of them quit smoking by the time of hospitalization. Patients who reported the highest tobacco consumption developed mucositis Grades 0 and I. Patients who reported low tobacco consumption presented mucositis Grade II. in the worst case scenario.

We observed that patients who developed mucositis Grade I rapidly evolved to mucositis Grade II and had a worse prognosis for oral mucosal healing compared with patients who presented Grade II mucositis over a longer period of time.

\section{Discussion}

According to Suresh et al., ${ }^{3}$ leukocyte levels below $3,000 / \mu \mathrm{L}$ increase the risk of oral mucositis occurrence, which is further increased in combination with factors such as poor oral hygiene and alcohol and tobacco consumption. In the present study, we did not observe a pattern of mucositis occurrence in patients undergoing HSCT because the degree of mucositis severity was not associated with the counting of leukocytes.

Some authors claim that young patients are more prone to severe mucositis, ${ }^{6}$ while others have reported that elderly patients are at greater risk of a high degree of mucositis. ${ }^{7}$ In this study, it was not possible to differentiate the degrees of mucositis among ages, and patients under 35 years of age (29\%) showed mucositis at Grades I, II, and III. One should take into account that pediatric treatment is not performed at the HSCT Department of the Hospital Erasto Gaertner; thus, the cohort of pediatric patients is reduced.

While several authors have suggested that satisfactory oral hygiene can prevent and treat oral mucositis in oncologic patients, ${ }^{8,9,10}$ this study did not find a relationship between the presence of biofilm and the occurrence of mucositis.

Chlorhexidine gluconate $0.12 \%$ is an antimicrobial agent widely used for oral hygiene. Some studies have proposed that the use of chlorhexidine mouthwash could decrease the severity of clinical symptoms of mucositis. ${ }^{11,12}$ The Multinational Association for Supportive Care in Cancer (MASCC) reported that chlorhexidine had no preventive effect on mucositis associated with irradiated head and neck cancer, while results with other types of treatment were not in agreement. ${ }^{11}$ Nonethe- 


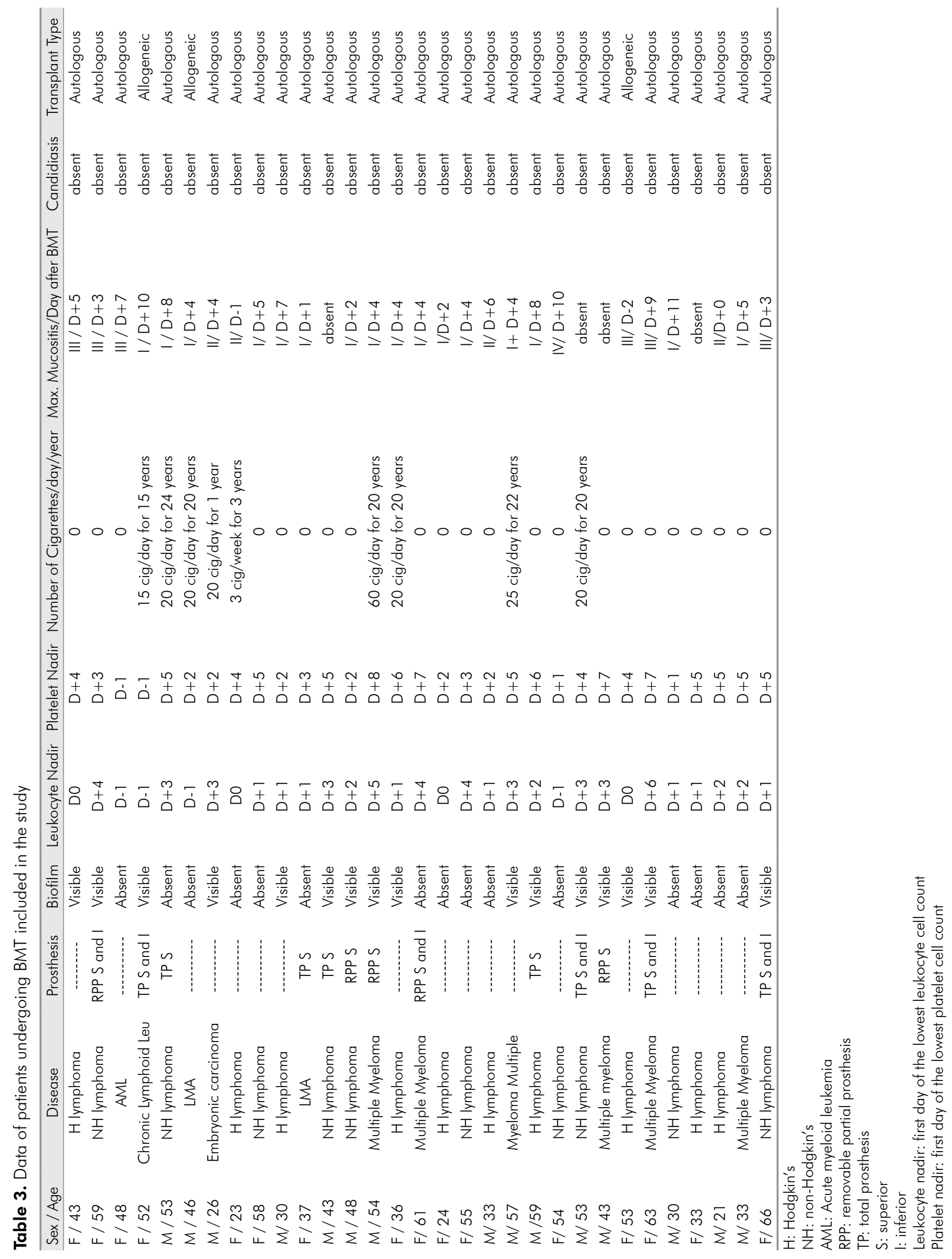


less, chlorhexidine mouthwash can be used for biofilm control, gingivitis, and other periodontal problems. ${ }^{11}$ Our department recommends chlorhexidine mouthwash as part of a protocol to improve oral hygiene.

The indirect effect of chemotherapeutic drugs occurs when bone marrow function is suppressed during the nadir of treatment, with a concomitant decrease in platelet and leukocyte levels. In addition, immunosuppression induced by treatment leads to increased susceptibility to infection and stomatotoxicity. ${ }^{13}$ Patients with Hodgkin's lymphoma received medication with high inflammatory potential (etoposide), and patients with non-Hodgkin's lymphoma received two drugs with high potential to cause mucositis, i.e., etoposide and cytarabine. The other patients did not receive medications with a high potential for mucosal inflammation. No difference in the incidence of mucositis was observed among HSCT protocols, even with the use of high mucosal toxicity drugs (Chart). Rapid mucositis progression was considered a poor prognostic factor during hospitalization. Mucosal cells have a 3- to 5-day half-life, resulting in epithelial renewal every seven to 14 days. ${ }^{14}$ The cytotoxic effects of chemotherapy interfere with cell proliferation and renewal and increase regeneration time, favoring the progression of mucositis. Patients who showed no mucositis had a different clinical history, with no correlation with nadir curves.

Several treatments for oral mucositis are proposed in HSCT centers and are described in the literature, but some are controversial and need further studies to prove their efficacy. ${ }^{15}$

Low-frequency laser therapy (LFLT) is a treatment used to decrease the severity of mucositis, reduce the size of the affected area, delay the occurrence of ulcers, and improve healing. ${ }^{16}$ Because our department started a protocol for the use of LFLT in all HSCT patients, we observed an important improvement in the patients' quality of life and a decrease in oral sensitivity, which is similar to that reported in the literature. ${ }^{8,14,15,16}$ In contrast to other reported results ${ }^{6,15}$ ( $15 \%$ of patients developed mucositis Grade IV), only three patients in our cohort presented severe mucositis.

Twelve patients used rehabilitative dental prostheses, and no differences in the degree of mucositis were observed in comparison with those who did not use prostheses. Removable dentures with good and satisfactory hygiene have been discussed in the literature as a preventive factor for oral mucositis; however, sometimes the use of such prostheses is discontinued. ${ }^{2,14}$ In this study, the decision to use oral prostheses during hospitalization was left to the patient; however, most of them reported difficulties and discomfort in the use of such prostheses.

Smokers were less likely to develop mucositis compared with nonsmokers. It was observed that high tobacco consumption was related to mild mucositis lesions, probably because of tobacco smoke-induced hyperkeratinization, which may have a protective effect on the mucosa ${ }^{14,17,18}$ (Table 4).

In addition, fungal infections are frequent in HSCT patients. ${ }^{19}$ As part of hospital protocol, all patients were prescribed antifungal prophylaxis during treatment; therefore, no clinical signs of oral candidiasis were observed, and comparison with chemotherapeutic nadir curves was not possible. Viral prophylaxis was performed for all patients during all treatments.

Chart. Chemotherapeutics with high potential to cause stomatitis ${ }^{18}$

\begin{tabular}{|c|c|c|c|}
\hline Antimetabolites & Drugs that Interact with DNA & Drugs that Interact with Tubulin & Drugs used in the BMT EGH \\
\hline $\begin{array}{l}\text { Cytarabine } \\
\text { 5-Fluorouracil } \\
\text { Floxuridine } \\
\text { Hydroxyurea } \\
\text { 6-Mercaptopurine } \\
\text { Methotrexate } \\
\text { 6-Thioguanine }\end{array}$ & $\begin{array}{c}\text { Actinomycin D } \\
\text { Amsacrine } \\
\text { Procarbazine Hydrochloride } \\
\text { Daunomycin } \\
\text { Doxorubicin } \\
\text { Etoposide } \\
\text { Idarubicin } \\
\text { Mitomycin C } \\
\text { Mitoxantrone } \\
\text { Mithramycin } \\
\text { Bleomycin sulfate }\end{array}$ & $\begin{array}{c}\text { Docetaxel } \\
\text { Paclitaxel } \\
\text { Vinblastine sulfate } \\
\text { Vincristine sulfate }\end{array}$ & $\begin{array}{c}\text { Busulfan } \\
\text { Carmustine } \\
\text { Carboplatin } \\
\text { Cyclophosphamide } \\
\text { Cytarabine } \\
\text { Etoposide } \\
\text { Filgrastim } \\
\text { Fludarabine } \\
\text { Melphalan } \\
\text { Mesna }\end{array}$ \\
\hline
\end{tabular}


Table 4. Former smokers and the presence of oral mucositis

\begin{tabular}{|c|c|c|c|c|}
\hline Sex/Age & Mucositis & Disease & Cigarette Use & Tobacco Intake \\
\hline$M / 53$ years & Grade 0 & NH lymphoma & 20 years, $20 \mathrm{cig} /$ day & High \\
\hline$M / 57$ years & Grade I & Multiple Myeloma & 22 years, $25 \mathrm{cig} /$ day & High \\
\hline $\mathrm{F} / 36$ years & Grade I & H lymphoma & 20 years, $20 \mathrm{cig} /$ day & High \\
\hline $\mathrm{F} / 52$ years & Grade I & Leukemia & 15 years, $15 \mathrm{cig} /$ day & High \\
\hline$M / 54$ years & Grade I & Multiple Myeloma & 20 years, $60 \mathrm{cig} /$ day & High \\
\hline$M / 53$ years & Grade I & NH lymphoma & 24 years, $20 \mathrm{cig} /$ day & High \\
\hline$M / 46$ years & Grade I & Leukemia & 20 years, $20 \mathrm{cig} /$ day & High \\
\hline $\mathrm{F} / 23$ years & Grade II & H lymphoma & 3 years, 3 cig/week & Low \\
\hline$M / 26$ years & Grade ॥ & Embryonic Carcinoma & 1 year, 20 cig/day & Low \\
\hline
\end{tabular}

Some authors have suggested that there is a slight increase in the incidence and severity of oral mucositis in patients undergoing allogeneic compared with autologous transplants. ${ }^{20}$ This may be due to medications that can induce mucositis. Our cohort had only three patients who underwent allogeneic transplant; therefore, we could not assess the difference between the two groups. However, several variables must be considered relative to HSCT for the occurrence of mucositis, and the type of transplant is only one of them. The present study had a fairly heterogeneous sample of patients with different ages, different diagnoses, risk factors, and other complex systemic conditions that directly affect the oral cavity.

\section{References}

1. Epstein JB, Shubert MM. Oral mucositis in myelosuppressive cancer therapy. Oral Surg Oral Med Oral Pathol Oral Radiol Endod. 1999 Sep;88(3):273-6.

2. Stramandinoli RT, Souza PHC, Wsetphalen FH, Bisinelli JC, Ignácio SA, Yurgel LS. Prevalence of oral candidiasis in hospitalized patients and evaluation of risk factors. Rev Sul-Bras Odontol. 2010;7(1):66-72. Portuguese.

3. Suresh AV, Varma PP, Sinha S, Deepika S, Raman R, Srinivasan $\mathrm{M}$, et al. Risk-scoring system for predicting mucositis in patients of head and neck cancer receiving concurrent chemoradiotherapy [rssm-hn]. J Cancer Res Ther. 2010 Oct-Dec;6(4):448-51

4. Blijlevens NM, Donnelly JP, Meis JF, De Keizer MH, De Pauw BE. Procalcitonin does not discriminate infection from inflammation after allogeneic bone marrow transplantation. Clin Diagn Lab Immunol. 2000 Nov;7(6):889-92.

5. World Health Organisaton. Handboock for reporting results for cancer treatment. Geneve: WHO; 1979.

6. Pico JL, Avila-Garavito A, Naccache. Mucositis: its occurrence, consequences, and treatment in oncology setting. Oncologist. 1998 Dec;3(6):446-51.

\section{Conclusion}

Oral mucositis is an important complication in cancer treatment involving HSCT. The prognosis for and treatment of this condition are uncertain and unpredictable. Methods of prevention must be elucidated. Our study found no relationship between the chemotherapeutic nadir curve and the occurrence of mucositis and the appearance of candidiasis. Thus, hematologic alterations that occur during chemotherapy do not appear to predispose oral mucositis or oral candidiasis. Furthermore, the use of LFLT can be an important technique in combatting mucositis, thereby decreasing the severity of this complication during the treatment process.

7. Devaraju CJ, Lokanatha D, Bapsy PP, Suresch AV, Viswanath G, Sandhya B. Risc Scoring for predicting Mucositis in Indian patients with esophageal carcinoma receiving concurrent chemoradiotherapy. Gastrointest Cancer Res. 2009 Jan-Feb;3(1):4-6.

8. Bonan PRF, Lopes MA, Alves FA, Almeida OP. Clinical, biological, histological features and treatment of oral mucositis induced by radiation therapy: a literature review. Rev Bras Cancerol. 2005;51(3):235-42. Portuguese.

9. Wohlschlaeger MSN. Prevention and treatment of mucositis: a guide for nurses. J Pediatr Oncol Nurs. 2004 Set-Oct;21(5):281-7.

10. Mendonça EF, Silva CLS, Silva JB, Palmeira CM, Silva GBL. Oral complications of chemotheraty and radiotherapy in cancer treatment. Rev ABO Nac. 2005;13(3):151-7. Portuguese.

11. McGuire DB, Fulton JS, Park J, Brown CG, Correa ME, Eilers J, et al. Systematic review of basic oral care for the management of oral mucositis in cancer patients. Support Care Cancer. 2013 Nov;21(11):3165-77 
12. Godim FM, Gomes IP, Firmino F. Prevention and treatment of oral mucositis. Rev Enferm UERJ. 2010;18(1):67-74. Portuguese.

13. Bultzingslowen I, Brennan MT, Spijkervet FK, Logan R, Stringer A, Raber-Durlacher JE, et al. Growth factors and cytokines in the prevention and treatment of oral and gastrointestinal mucositis. Support Care Cancer. 2006 Jun;14(6):519-27. Epub 2006 Apr 21.

14. Pinho AP, Misorelli JC, Montelli R, Longato SE. Mucositis related-cancer treatment. Sci Health. 2010;1(3):145-60. Portuguese.

15. Eduardo FP, Bezinelli LM, Hamerschlak N, Andrade CT, Morelli LR, Corrêa L. Oral care in Brazilian bone marrow transplant centers. Rev Bras Hematol Hemoter. 2011;33(1):15-20.

16. Antunes SH, Azevedo AL, Bouzas LSF, Adão CA, Pinheiro $\mathrm{CT}$, Mayhe R, et al. Low-power laser in the prevention of induced oral mucositis in bone marrow transplantation patients: a randomized trial. Blood. 2007;109(5):2250-5.
17. McRobbie H, Hajek P, Gillison F. The relationship between smoking cessation and mouth ulcers. Nicotine Tob Res. 2004 Aug;6(4):655-9.

18. Floto RA, Smith KG. The vagus nerve, macrophages, and nicotine. Lancet. 2003 Mar 29;361(9363):1069-70.

19. Castro CG, Grefianin LJ, Brunetto AL. Bone marrow transplantation and cord blood transplantation in children. J Pediatr (Rio J). 2001 Sep-Oct;77(5):345-60. Portuguese.

20. Cutler C, Li S, Kim HT, Laglenne P, Szeto KC, Hoffmeister $\mathrm{L}$, et al. Mucositis after allogeneic hematopoietic stem cell transplantation: a cohort study of methotrexate- and non-methotrexate-containing graft-versus-host disease prophylaxis regimens. Biol Blood Marrow Transplant. 2005 May;11(5):383-8. 